\title{
CIENCIA, IDEOLOGIA Y POLITICA EN EVALUACION DE PROGRAMAS
}

\author{
Rocio Fernández Ballesteros \\ Universidad Autónoma de Madrid (1)
}

Es difícil comenzar una serie de conferencias sobre un título general - que, en forma abreviada, va a ser el de evaluación de programas - sin descender a cuestiones de definición y objeto.

Voltaire aconsejaba: "definid los términos que empleáis" y ello parece una sana costumbre al versar sobre cualquier cuestion. Sin embargo, se supone que las personas congregadas aquí, no sólo están interesadas en el tema sino que, por su formación, tienen - en mayor o menor medida - conocimiento sobre él. A pesar de ello, ya que los conceptos pueden tener diferentes significados y con el fin de homologar un vocabulario básico, no parece baladí la tarea de plantear, inicialmente, algunas referencias conceptuales.

Como todos ustedes saben, durante los años 60 y 70 comenzaron a realizarse - en estados que habían al canzado un alto grado de desarrollo económico - una serie de reformas sociales con el fin general de mejorar la calidad de vida del ciudadano en el llamado "estado de bienestar" y con la meta de llegar a "una nueva frontera". Estos conceptos, inspirados en ideas post-keynesianas fueron objeto de duras críticas a partir, tanto de los movimientos contraculturales de los años 70 como de la crisis económica de los 70 . Todo ello no mermó la puesta en práctica de tales objetivos, los cuales llevaron consigo acciones de muy distinta índole (educativas, sanitarias, ambientales, etc.), ejecutándose a través de un determinado proceso de planificación. Pues bien, con la triple meta de completar una programación por objetivos, proceder al control del gasto público y mejorar la calidad de las acciones emprendidas, se ha ido constituyendo durante los últimos 20 años una nueva disciplina o un nuevo campo de acción del científico social. Esta disciplina, fundamentalmente aplicada, ha ido adoptando diversos nombres: "investigación valorativa", "estudios valorativos" o la rúbrica que aqui se ha adoptado, "evaluación de programas". Todos estos términos, claramente intercambiables entre sf, hacen referencia - en definición de Rossi y Freeman (1985) - a la sistemática aplicación de los procedimientos de investigación social al enjuiciamiento o análisis sobre el mérito, valor o eficacia de concretas acciones humanas implantadas en la realidad social. Pero, tales acciones, a su vez, se 
realizan coordinadamente dentro de una "política" concreta, ejecutada a distintos niveles de complejidad a través de "programas", "servicios", "tratamientos" o "intervenciones". Como señalan Berk y Rossi (1976), es fácil aceptar que tales apelativos hacen referencia a cualquier actividad organizada dispensada generalmente por el sector público con la finalidad de mejorar la calidad de vida de los ciudadanos. $\mathrm{O}$, como señala Cook (1984), un programa es un conjunto heterogéneo de acciones, a veces, con una difusa estructura, cuyo objetivo es el de resolver algún problema que incumbe a los ciudadanos. Pero, los problemas de una sociedad son de muy variado tipo, asi que la pluralidad de objetivos de los programas a los que me refiero se ve recortada a determinados ámbitos.

Un análisis de una de las publicaciones más importante sobre este tema (Evaluation Studies. Annual Review) puede dar cuenta de esta cuestión. Así, teniendo en cuenta la última década, los trabajos publicados versan sobre los siguientes temas:

- un $32 \%$ sobre cuestiones conceptuales y metodologicas

- un $25 \%$ sobre evaluación de programas de salud

- un $21 \%$ sobre valoración de programas educativos

- un $10 \%$ al análisis del funcionamiento de servicios sociales

- finalmente, un $12 \%$ a la evaluación sobre decisiones legislativas, ambientales y otros.

En resumidas cuentas, que la evaluación a la que me refiero concierne, fundamentalmente, al enjuiciamiento sobre el valor de acciones emprendidas para mejorar la educación, la salud y las prestaciones sociales en diversos campos. Esta es la razón que justifica la presencia en estos días de personas con responsabilidad política en la evaluación de estos tipos de programas.

Pero, como decía, un programa cuenta con distintos niveles de complejidad y distintos grados de implantación. Es decir, por poner un ejemplo, el propósito o política general de la "promoción de la salud" presente en la ley general de sanidad, ha dado lugar en España a una serie de acciones y servicios de muy distintos niveles de complejidad, los cuales han sido implantados en las distintas administraciones del Estado (estatal, autonómica, provincial y local). Por tanto, la evaluación se tendrá que dirigir - según su complejidad y amplitud, y utilizando la terminología de Cook y Shadish (1986) - a un concreto Programa, Proyecto o Elemento. Poniendo un sencillo ejemplo, uno de los programas que vehicula la promoción de la salud comprende distintos servicios implantados como proyectos (a veces no bien coordinados) a 
distintos niveles, o en distintos sectores, de la administración del estado. Estos servicios dispensan distintos elementos o intervenciones y tratamientos específicos; por ejemplo, prevención buco-dental, atención domiciliaria al anciano, etc.

Explico esto porque espero que desde un principio quede claro que durante esta semana no vamos a tratar aqui sobre la evaluacion de "pol fícas" realizadas para una mayor bienestar social sino de concretas acciones implantadas al servicio de concretas "políticas" con ese estimable - y , a su vez, valorable - propósito: el bienestar social.

Pero, la evaluación -semántica y terminológicamente - hace referencia al establecimiento del "valor" o "mérito" de un determinado objeto, en nuestro caso - como decfa - un programa, proyecto o elemento. Como todos ustedes saben, existen múltiples criterios con el fin de establecer lo "valorable" de cualquier acción humana. Imaginense la dificultad de llegar a una homologación o estándar sobre cómo juzgar el mérito de un concreto programa de acción social. Me atrevería a decir, en términos hiperbólicos, que hablar del valor de un programa es como filosofar sobre la bondad del hombre. No existen criterios fijos, predeterminados. De forma tal que la evaluación pueda realizarse en función de si el programa es pertinente, es decir, es potencialmente útil en la resolución del problema implicado o si ha sido suficientemente diseñado o si es eficaz en el sentido de haber alcanzado los objetivos propuestos o eficiente, según análisis costo-beneficio. Y ello por citar algunas de las posibles alternativas.

El último extremo de la definición que inicialmente daba, hacía referencia al cómo se realiza la evaluación y decla que se aplicaban los procedimientos comunes en investigacion social. Ello hace referencia a que son procedimientos científicos los que están comprometidos en el hacer común de la evaluación de programas, por lo que ésta también se verá concernida por los problemas generados desde el ámbito científico. Por ello, durante estos días eminentes profesionales de la evaluación van a referirse a problemas metodológicos y conceptuales relativos a distintos aspectos del quehacer científico de la evaluación.

Quisiera terminar estos primeros conceptos introductorios resaltando la importancia que en los últimos años ha alcanzado la evaluación de programas. Algunos de los indicadores de esto que digo son los siguientes: institucionalización de esta actividad en distintos parses europeos y norteamericanos, creación de sociedades científicas, órganos de difusión, agencias públicas y privadas dedicadas a este menester, grandes sumas invertidas e institucionalización - en algunas administra- 
ciones del estado - del rol de evaluador de programas. En todo caso, la mejor prueba de lo que digo es la presencia aquí de una audiencia tan numerosa. Mi fundamental objetivo hoy es poner de relieve la complejidad que conlleva la evaluación de programas, su alcance y, fundamentalmente, las implicaciones en distintos órdenes que cualquier acción evaluadora conlleva y que traspasa, con mucho, la pura investigación cientifica considerada ésta - si ello fuera posible - como una actividad aséptica, libre de toda influencia ideologica o política.

\section{Ciencia, ideología y política en el proceso de planificación social}

He dicho también antes que, de fondo, la evaluación de programas surgió de una clara conciencia de planificación de las acciones a implantar. Ello implica, por tanto, que la evaluación forma parte del ciclo de toma de decisiones que toda programación social lleva consigo. Ciclo o proceso, a través del cual se pretende resolver problemas que, si bien son de distinta índole (sanitarios, educativos, de asistencia social, ambientales), todos ellos tienen el denominador común de ocurrir en una realidad sociopolítica y pretender actuar sobre la calidad de vida de los habitantes de un específico contexto.

\section{Esquema 1}

EL LUGAR DE LA EVALUACION EN UN CICLO DE TOMA DE DECISIONES

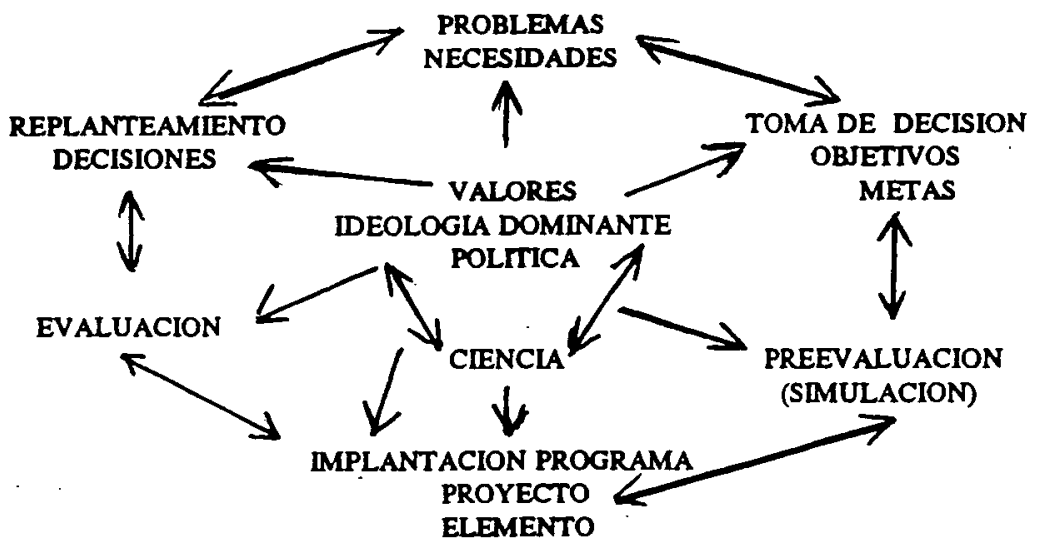

En este esquema he tratado de plasmar los pasos fundamentales de este proceso. En un primer momento se focaliza el problema que se 
pretende resolver, el cual lleva consigo una serie de necesidades en la población. Para llegar a resolver dicho problema se suelen plantear una serie de objetivos, los cuales se especifican en metas a alcanzar. Con el fin de alcanzar esas metas se seleccionan una serie de acciones, programas, proyectos o tratamientos. Tras su implantación en un determinado contexto, se procede a su evaluación. El análisis de resultados va a permitir tomar nuevamente decisiones: apoyar la continuación del programa, su modificación o supresión. Vemos cómo, en ese ciclo, la evaluación de resultados permite la comprobación de la eficacia y eficiencia de un programa siendo un eslabón más de un amplio conjunto de toma de decisiones polfiticas.

Pero ya he dicho que existen varios tipos de evaluación según la función que desempeñe y los distintos criterios utilizados a la hora de enjuiciar un programa de acción social. Amén de la evaluación sumativa o de resultados, la claramente visible en el esquema, también se puede proceder a la valoración de cómo se está llevando a cabo la implantación del programa con el fin de ir mejorándolo, realizando entonces una evaluación del proceso o formativa. O realizando una evaluación (o preevaluación) sobre si los medios y acciones a emprender van a permitir la consecución de los objetivos propuestos. O, cómo no, la evaluación puede ir asociada a todo lo largo del proceso de toma de decisiones, evaluando empíricamente necesidades, evaluando si los objetivos propuestos son realistas y en consonancia con las necesidades o, como ocurre en la evalucación valorativa, si en un programa se han puesto a contribución los medios necesarios para que éste sea evaluado.

Lo más importante es resaltar que el proceso de planificación y evaluación de programas se produce, logicamente, en un contexto histórico, político, ideológico y cientifico y que todos y cada uno de los momentos del ciclo de toma de decisiones se ve impregnado de estos componentes del contexto.

Tenemos que tener en cuenta, como señala Parsons, que la función básica de cualquier ideología es la de definir un programa particular de acción social como merecedor y digno de apoyo. Ninguna política, como ningún programa, puede estar libre de planteamientos ideológicos. Con lo cual, por supuesto, no hace sino referirse a la función legitimadora de la ideología ya sugerida por Marx y formulada explfitamente por Mannheim, entre otros.

Desde luego, en países democráticos, los programas políticos de los partidos presentan explícitamente su ideología de base en la que se fundan el análisis de los problemas fundamentales de una colectividad 
y las acciones que van a ser emprendidas -de lograr la confianza de la mayoría- en orden a la resolución de las supuestas necesidades existentes. La influencia que los presupuestos ideológicos y políticos, asf como (en palabras de Rosch) las teorfas ingenuas que la gente tiene, en el ańlisis y jerarquización de los problemas existentes en una colectividad determinan no solo la forma en la cual se tratan de resolver sino, como derivación lógica, los objetivos que se pretenden alcanzar. Pero, a veces, los problemas no se priorizan adecuadamente; en ocasiones se implantan acciones de incidencia mínima, como cuando se realiz 6 una determinada actividad con un importante soporte económico con no más de una decena de usuarios (hijos nacidos de madres en prisión), y esto es tan sólo una anécdota. $O$, también, a veces los objetivos a través de los cuales se juzga un programa son modificados sobre la marcha, en algunos de los proyectos que lo vehiculizan. Asf, por ejemplo, en Venezuela la aplicación del entrenamiento cognitivo "desarrollo del pensamiento", como un elemento más del Proyecto implantado en el ámbito laboral, en conexión con los Programas para el desarrollo de la inteligencia, fue evaluado en una concreta Empresa (subvencionada para ello por el Estado), en función de una menor conflictividad laboral. De ello nada sabran los responsables gubernamentales promotores de los programas cuyo objetivo era el desarrollo cognitivo del pueblo venezolano. $O$, muchas veces se implantaban acciones para las que se requiere una preparación en el usuario, sin la cual el programa fracasará. Así, en un pueblecito extremeño un al to porcentaje de los ciudadanos beben agua de pozo desde que el poder municipal implantó la medida sanitaria de clorar el agua corriente, ya que existe la falsa creencia -o la teoría ingenua- de que la cloración es perjudicial. Modificar la creencia hubiera sido necesario antes de aplicar la medida.

En definitiva, los programas sociales raramente exceden los límites de la ideologfa dominante y ésta afecta también a las definiciones de los criterios valorativos. En otras palabras, la evaluación puede validar una particular visión y solución de un problema social enfatizando ciertos resultados como opuestos a otros, sirviendo a fines de autocomplacencia política. Pero, además los medios -acciones, tratamientos, etc.- seleccionados a la hora de conseguir los objetivos propuestos se ven también impregnados por una cierta carga ideológica. Pongamos un ejemplo, tratando de simplificar el tema, y como señala Seidman (1983), a la hora de abordar el problema de la delincuencia (inicialmente considerado por su resonancia social en encuestas o mediante el análisis de indicadores objetivos, número de acciones a 
través de las que se infringe el código penal) e implantar acciones sociales, suelen exigir explicítamente o implicitamente distintas teorías que revisten una carga ideológica, en el sentido de estar integradas dentro de un modelo de creencias morales y cognitivas sobre el hombre. La delincuencia puede ser entendida como una derivación del paro, como un producto de una legislación inadecuada, como un resultado del incremento de la droga, como una enfermedad genética, como un producto del proceso de socialización. No cabe duda de que, con independencia de los resultados científicos, existen bases ideologicas en las distintas concepciones de este tipo de comportamiento antisocial. Tampoco debe caber duda de que los programas elegidos estarán basados en hallazgos cientificos que avalen la ideologia dominante.

Pero, además, la implantación de programas no sólo tiene como relevantes, a la hora de su evaluación, parámetros o dimensiones físicas y sociales. El tiempo es una dimensión de extraordinaria importancia. Un programa se aplica durante un período de tiempo determinado, en ese intervalo de tiempo suelen ocurrir múltiples eventos que influyen en mayor o menor medida en los resultados que de él se obtienen. El tiempo, también introduce todo tipo de variables contaminadoras a las que me referiré mas adelante. Pero además, un programa requiere, para cumplir sus objetivos, su aplicación durante un intervalo generalmente dilatado. Ello es relevante por cuanto sucede con programas sociales, o de otro tipo, se ven ligados a concretar políticas y al existir una alternancia en el poder se produce un constante tejer y destejer en la planificación social con los riesgos y costos que eso conlleva. El periodo legislativo (de un fátidico $5+6-1$ ) lleva en ocasiones, a los responsables de la politica social, a implantar acciones apresuradamente. Ni que decir tiene que muchos de los problemas existentes, como muchos de los objetivos planteados, requieren acciones prolongadas para prolongar sus efectos benéficos. Son también cuestiones constitucionales las que pueden determinar aparentes fracasos. Asi, en Venezuela, los programas para el desarrollo de la inteligencia -en los cuales se invirtió una gran cantidad de energía y esfuerzos económicos- fueron aplicados el tiempo que duró la estancia en poder del partido proponente. Ya que muchos de los proyectos implantados requerían una implantación prolongada, con el fin de poder obtener resultados concluyentes, la experiencia fue abortada. Digo esto, con independencia de si los objetivos del Ministerio para el desarrollo de la Inteligencia fueran coherentes con las necesidades y problemas de los venezolanos.

Pero, además, como señalan Cook y Shadish (1986), la evalua- 
ción es, desde distintos puntos de vista, un acto político que ocurre en un contexto donde el poder, la ideología y los intereses son, seguramente, más poderosos que los resultados de las propias evaluaciones. En otros términos, la propia decisión de evaluar un programa está también "contaminada" en el sentido de no verse libre de influencias sociopolfticas. Desde el punto y hora de la importancia que tenga para el político la valoración "científica" de su actividad, al poder económico que implica la contrastación de los servicios del evaluador, nada de ello se ve libre de tales influencias. Por último, las repercusiones de la evaluación que, teóricamente, deberán influir en la eliminación o mejora del programa, como luego trataré de demostrar, también están impregnadas de estos componentes.A este extremo espero se dedique la Mesa redonda que tendrá lugar el miércoles por la tarde.

En conclusión, si pretendo dar cuenta del complejo título de mi intervención será necesario examinar, al menos, las siguientes cuestiones: Relaciones entre Ciencia y Evaluación de programas, entre Ciencia y Política asf como, realizar la difícil tarea de sondear las Ideologías en la Evaluación.

La propia definición de evaluación de programas de la que he partido hace referencia a que esta disciplina supone una tecnologla derivada de los procedimientos de investigación social y si analizásemos la mayor parte de los trabajos metodológicos existentes, creo que habría que especificar diciendo que en consonancia con la metodología científica, entendiendo por tal aquella forma de hacer ciencia acuñada desde el positivismo, sobre todo por sus repercusiones en las ciencias sociales. En los últimos años han ido desarrollándose -no sé bien si se puede decir que en paralelo- dos formas de hacer investigación social. Así que dos han sido también los modelos adoptados en evaluación de programas: el positivista y el naturalista. Ambos sostienen asunciones ontologicas, epistemológicas y metodológicas diferentes. En el cuadro siguiente pueden verse algunos de los presupuestos o sistemas de creencias básicos de ambos modelos.

Así, como principal asunción ontológica, desde plantemientos positivistas, el mundo externo existe como realidad independiente del perceptor $y$, por tanto, del investigador. Contrariamente, desde una postura naturalista existen múltiples realidades socialmente construidas.

Desde una perspectiva epistemológica, desde el positivismo, se mantiene que puede darse un conocimiento objetivo de la realidad, mientras que desde una posición naturalista la posibilidad única es la de 
un conocimiento subjetivo.

Por último, metodológicamente, desde el positivismo se enfatiza la importancia de la manipulación, la medición y el control mientras que desde un posicionamiento naturalista se proponen métodos supuestamente no invasivos, considerando que es el evaluador el instrumento de análisis por excelencia.

\section{Cuadro 1}

\section{ALGUNOS PRESUPUESTOS DE LA EVALUACION DE PROGRA- MAS POSITIVISTA Y NATURALISTA}

\section{POSITIVISMO}

\section{REALISMO}

Existe una realidad independiente de cualquier observador en la que rigen principios. La verdad es definida como la representación de la realidad.

\section{OBJETIVISMO}

El conocimiento de la realidad puede darse con independencia del observador en tanto en cuanto existen una serie de garantías de la observación.

\section{INTERVENCIONISMO}

El control, la manipulación y la medición son garantías necesarias para la investigación científica.

\section{NATURALISMO}

\section{RELATIVISMO}

Existen varias realidades socialmente construidas en las que no rigen principios ni leyes generales. La verdad es una construcción consensuada.

\section{SUBJETIVISMO}

La relación entre el observador y lo observado es de tal naturaleza que el producto de la investigación es una pura creación de ésta.

\section{HERMENEUTICA}

La metodologia compromete al observador que se convierte en el principal instrumento en la comprensión del fenómeno objeto de estudio.

Basado en Guba (1987)

Con base en estos supuestos básicos, Guba (1987) propone una serie de teoremas que se presentan en el cuadro 2. 
Cuadro 2

\section{TEOREMAS POSITIVISTA Y NATURALISTA EN EVALUACION (Guba, 1987)}

\section{POSITIVISTA}

1. La evaluación es una forma de investigación cientffica.

2. La evaluación produce datos contaminados por valores. Los valores distorsionan los datos cientificos.

3. Costos y responsabilidades pueden siempre ser determinados por cadenas de causa-efecto.

4. Los evaluadores pueden encontrar una posición que les permita la objetividad necesaria para sus actividades.

5. Los evaluadores son los canales de comunicacion a través de los cuales los verdaderos datos son pasados a las audiencias en los informes de valoración.

6. La valoración científica está especialmente legitimida y tiene un status especial que le confiere prioridad sobre otras conideraciones.

\section{NATURALISTA}

1. La evaluación es una forma de investigación naturalista

2. La evaluación produce datos en los cuales los "hechos" y "valores" están ligados inexorablemente. Los valores forman parte de la evaluación, son la base de atribución de significado.

3. Costos y responsabilidades son características de conglomerados de mutuas y simultáneas impresiones. Ninguna de ellas separadamente puede alabar o criticar.

4. Los evaluadores son compañeros subjetivos de los implicados en la creación de los datos valorativos.

5. Los evaluadores son orquestadores de su proceso de negociación, que trata de culminar en el consenso del mejor informe de las más sofisticadas construcciones.

6. La valoración naturalista no tiene ningún status ni legitimación especial, simplemente representa otra construcción más que será tenida en cuenta en la búsqueda del consenso. 
De ellos quisiera destacar dos:

1) Que mientras desde una metodología cientifica positivista se supone que valores y creencias distorsionan los datos obtenidos, desde el modelo naturalista los valores son considerados una parte intrínseca al proceso de evaluación e, incluso, proveen de significado a la evaluación, y

2) Que mientras los datos de una evaluación cientifica están especialmente legitimados y cuentan con un especial status y prioridad frente a cualquier otra consideración evaluativa, los datos procedentes de una investigación naturalista representan otra construcción más que será tenida en cuenta entre todas las posiblemente existentes en el contexto de investigacion.

He tratado de presentar ambos modelos de investigación lo más objetivamente posible. Al así hacerlo, me he decantado por un modo de hacer evaluación que trata (al menos lo intenta) de ser objetivo (en el sentido subjetivo) y que utiliza el método científico-positivo.

Lo que está claro, como señala Guba, es que en las dos formas de hacer evaluación existe un sistema de creencias apriorístico, sin posibilidad de contrastación, que es irreconciliable entre los dos modelos. No conviene confundir esta polémica entre los modelos positivista y naturalista con la existente entre métodos cualitativos vs cuantitativos. Mientras que la primera de ellas supone asunciones incompatibles entre ambos, la segunda permite la síntesis e integración tecnológica de indudables ventajas para una más completa evaluación.

Pero no es el momento de plantear cuestiones filosoficas de fondo -aún apasionantes- en las que no tenemos tiempo de detenernos. Tan sólo una puntualización, por si ustedes desean entrar más tarde en debate.

Desde una gnosología evolutiva se plantea, no sin pruebas, que el método cientifico, como fuente de conocimiento, es un eslabón filogénetico más, supone un mecanismo adaptativo resultado del proceso evolutivo cognoscitivo humano conducente a la verdad objetiva. Según epistemólogos como Oeser (1984) o biólogos como Lorenz, el conocimiento obtenido mediante el método cientifico es diferente del "primo aspecto" del conocimiento cotidiano y está sujeto a los principios básicos evolutivos, como aś lo es la ley básica biogenética de Haeckel.

Dicho esto, lo que sí es cierto es que el positivismo lógico ha entrado en una importante crisis, como así sostienen la mayor parte de los epistemológos. La cuestión es, como señala Brown (1984), que mientras algunos realizan aportaciones no rupturistas y se siguen considerando positivistas renovados, otros consideran el sistema ago- 
tado y proponen una nueva epistemología de la ciencia. En todo caso, la investigación cienfífica es tarea ardua y compleja y no cabe duda de que sugerencias metodológicas como la de Rowan, Harré o Secord (véase por ejemplo Reason y Rowan, 1981) alivian la tarea extraordinariamente, y lo digo, por si no se ha notado, malintencionadamente.

¿Cómo se resuelve esta situación en evaluación de programas?. Autores como Campbell, Glaser, Rossi, o incluso Scriven, están planteando una aproximación, que algunos llaman, "postpositivista" (que, personalmente, creo perfectamente compatible con los presupuestos fundamentales del positivismo) que Cook (1985) ha conceptualizado bajo la rúbrica de multiplismo. Diez son los puntos básicos de este positivismo renovado, aplicables a la evaluación de programas:

1. Establecer múltiples definiciones operacionales,

2. Utilizar múltiples métodos de evaluación,

3. Establecer programas basados en múltiples estudios interco nectados.

4. Realizar síntesis de estudios múltiples independientes (metaanálisis).

5. Construir modelos causales multivariados,

6. Plantear múltiples hipótesis rivales,

7. Interrogarse sobre cuestiones valorativas procedentes de todo tipo de personas implicadas en los programas,

8. Utilizar múltiples enfoques teoricos,

9. Realizar múltiples análisis de datos,

10. Establecer objetivos múltiples que permitan un heterogéneo análisis de resultados.

En definitiva, este decálogo propuesto por Cook -a la vez que supera la mayor parte de las críticas al empirismo lógico -enrianece la evaluación de programas. La única amenaza reside en que, por $l_{i \text {; propia }}$ complejidad que conlleva, pudiera hacer inviable la investigación.

Pero está claro que la evaluación de programas no sólo supone una aplicación de la metodologia de investigación social en el enjuiciamiento sobre el valor de algunas acciones humanas a las que, genéricamente, hemos llamado programas. Ya ha dicho que éstos, se basan en concretas teorias procedentes de la investigación básica. Como ya señalaba Merten (1973/1985) en su trabajo de 1949, pionero en estas cuestiones, i "Dimensiones técnicas y morales de la investigación de políticas"), "la teoría básica abarca conceptos fundamentales (variables 
y constantes), amén de postulados, teoremas y leyes. La ciencia aplicada, y en este caso, la evaluación de programas, consiste simplemente en discernir sobre cuestiones previamente probadas" (Merton, p. 151). Así, no se plantea una campaña de prevención contra el SIDA si no se tuvieran una serie de conocimientos científicos sobre cómo se contrae la enfermedad. Ni se aplicarian programas de higiene bucodental en las escuelas si no fuera porque se conoce la influencia benéfica de ciertas prácticas en el número de caries dentales. Pero, en muchas ocasiones, las cosas no son tan sencillas como sugieren ambos ejemplos. A veces, los programas se basan en supuestos teoricos, no suficientemente contrastados, para los que existen una o varias teorfas alternativas posibles y, en la mayor parte de las ocasiones, el programador social no se plantea los supuestos subyacentes al programa. Es por esto por lo que, cada vez con más fuerza, se está reivindicando la evaluación de las teorfas de base de los programas objeto de evaluación. Como señala Bickman (1987), la teorla de un programa no es ni más ni menos que una construcción de un modelo de cómo y por qué un programa se supone va a funcionar $\mathrm{o}$ va a tener efectos positivos sobre las unidades a las que se le aplica. Desde este punto de vista, la evaluación de un programa conlleva la evaluación de la teoría que en él subyace. $Y$, desde luego, los resultados de una determinada evaluación podrían ser considerados como hallazgos cientificos básicos. Ciencia y evaluación de programas estarian asi infinitamente relacionados y la evaluación sería más que una pura tecnologia.

Recientemente Cook y Shadish (1986) se han referido a la existencia de tres enfoques generales (o ¿teorías?) de la evaluación:

1) Desde el primero de ellos (basada en los planteamientos de Campbell, 1969, 1971) se considera a la evaluación como una forma de identificar soluciones manipulables. Desde esta perspectiva, es más importante conocer cómo y por qué ciertas soluciones a los problemas sociales planteados son efectivas o funcionan que conocer hasta qué punto lo hacen. Es decir, para Campbell (como para Scriven), la valoración está el servicio del interés público más que al servicio de intereses de concretos grupos de implicados o responsables de los programas.

2) Por otra parte, otros evaluadores como Cronbach (1982), Rossi y Freeman (1985) y, seguramente Weiss $(1977,1978)$, presentan una alternativa en la que el objeto fundamental estriba en identificar explicaciones generalizables. Se asume que la generalizabilidad del conocimiento facilita su transferencia según la extensión en la que los 
problemas sociales son eventualmente mejorados.

3) Por último, investigadores sociales como Patton o Wholey, consideran como objetivo fundamental de la evaluación el proveer de un servicio a los implicados. Postulan estos autores que la meta última de la evaluación es la de mejorar los problemas sociales y ello ocurre sólo si los programas explícitamente se ajustan a las necesidades de los implicados (managers de los programas, responsables, usuarios), es decir, de aquéllos que tienen relaciones con los proyectos, programas o elementos que se evalúan.

Lo más importante para nuestros fines es que estos tres modelos planteados por Cook y Shadish están basados en la lógica de los objetivos de evaluación y ésta acerca al evaluador, en mayor o menor medida, a la Ciencia, en el primer y segundo modelo o a la Política, en el tercero. Es decir, en la medida en la cual nuestros objetivos fueren los de averiguar cómo se resuelven mejor los problemas sociales o cómo generalizar más adecuadamente los hallazgos obtenidos, estarfamos más cerca de considerar a la evaluación de programas al servicio de la Ciencia. Por otra parte, si tratásemos de probar si unas determinadas acciones políticas cumplen los objetivos politicos establecidos, la evaluación serf́a una tecnologf́a al servicio de de políticas. En definitiva, estarfamos frente a la distinción de Bickman (1980) entre "orientación teórica" y "orientación a los problemas"realizada para explicar el desarrollo de la ciencia, en este caso útil para diferenciar modelos de evaluación de programas.

En otras palabras, el evaluador puede servir a dos sectores -la ciencia y la política-a uno de ellos prioritariamente, o tratar de sintetizar ambas servidumbres. Esta última es la propuesta de Chen y Rossi (1982) cuando presentan un modelo de valoración integrando, una justificada base teorica de los programas junto a múltiples objetivos de evaluación que satisfaga a los implicados.

Sin embargo, no es conveniente ser demasiado optimistas suponiendo que los hallazgos evaluativos van a permitir la contrastación empírica de supuestos teóricos, ni siquiera cuando se realiza un trabajo riguroso. En definitiva, como señala Campbell (1969) la lógica de la inferencia indica que la investigación cientifica experimental (o cuasiexperimental) no permite "probar" ("prove") teorías, sino solo permite el hallazgo de "pruebas" sobre su bondad ("probe"). Como ya se ha dicho antes, y es la principal crítica al positivismo lógico, para cada teoría corroborada existe una infinidad de explicaciones rivales. Y, lo que es peor, la mayor parte de esas hipotesis alternativas suponen 
artefactos introducidos en la investigación por medio tanto de los propios tratamientos o programas como de los dispositivos de medida de sus efectos. Si esto se refiere a la ciencia social básica, la cuestión se agrava a la hora de hacer referencia a la aplicación de estos conocimientos a la realidad social. En primer lugar, porque existe una mayor equivocidad en la inferencia causal de la investigación realizada en "settings" socio-politicos; en otros términos, existe un mayor número de hipotesis rivales plausibles, existe un menor control de variables y la "naturalidad" (y la validez interna y externa) se ve comprometida por múltiples amenazas. $Y$ es que, como antes señalaba, el contexto sociohistórico suele jugar malas pasadas e introduce todo tipo de variables contaminadoras. Pondré un ejemplo -tomado de Cronbach (1982)- que puede ser de utilidad como ilustración de lo que digo. Procede de la valoración realizada en Guatemala sobre el programa alimenticio. Se trataba de saber si una dieta enriquecida dada a madres embarazadas y a sus hijos influra en un mejor desarrollo intelectual de éstos. Para averiguarlo se programó un diseño experimental. En cuatro lugares se emplearon centros de salud conectados con el programa. En dos de ellos, elegidos al azar, se proporcionaba el tratamiento elegido: es decir, se suministraba una papilla enriquecida a madres y niños. En los dos lugares control se administraba una bebida dulce con escaso valor nutritivo. Tras un determinado tiempo y a una determinada edad de los niños, deberían aplicarse unos tests de medida del desarrollo cognitivo. Como señala Cronbach "serfa erróneo considerar esta investigación como una comparación estadistica con varias decenas de casos por tratamiento. Cada aldea constitura un caso con su propia y continua historia. El hecho de promediar simplemente deja de lado algunos de los efectos más importantes obtenidos. Puesto que tomar la comida o la bebida era una actividad voluntaria, se dieron variaciones importantes dentro y entre las aldeas. La asistencia al Centro de Salud podría haber aumentado en aquéllos cuyo recepcionista fuera especialmente simpático. Una mayor frecuencia de asistencia no sólo significaba más comida sino mayor estimulación social e intelectual. Pero aún ocurrieron más eventos relevantes; de hecho, se inició la actividad guerrillera antigubernamental cerca de una de las aldeas, la asistencia al Centro disminuyo, puesto que cualquier contacto con organismos del Estado podia ser interpretado como un gesto de lealtad política. En las aldeas que proporcionaban la bebida refrescante, los chicos podrfan haber ingerido más que las chicas. Se sospechó que las mujeres que pedian más papilla enriquecida comían menos en casa con el fin de que sus maridos 
se alimentaran mejor. En los analisis estadísticos se analizó la cantidad de papilla que cada niño habra recibido, pero no lograron establecer los restantes factores intervinientes. Los investigadores guatemaltecos querfan medir el efecto de una sola variable causal, el incremento dietético sobre el desarrollo intelectual. Sin embargo, los resultados se vieron afectados irremisiblemente por innumerables variables y ya se sabe qué sucede cuando existen más variables que casos, cualquier inferencia se hace imposible.

Pueden ustedes imaginarse que este ejemplo me ha parecido pertinente con el fin no solo de plantear la complejidad de la investigación evaluativa, sino de ponderar todo lo hasta aquí dicho y subrayar las dificultades existentes al efectuar inferencias y generalizaciones procedentes de los resultados de la evaluación de programas. El contexto socio-histórico involucrado dificulta enormemente la obtención de conclusiones válidas. Sin embargo, creo que debemos ser optimistas, ya que es posible un análisis más ajustado de los realizados hasta ahora a partir de planteamientos multiplistas.

Bien, hasta aqur, las relaciones interactivas entre ciencia y evaluación. Veamos las existentes entre Ciencia y Política.

\section{Ciencia y Política}

Si he mantenido -siguiendo a epistemólogos evolutivos- que el método científico forma parte de la historia natural del conocimiento humano, nos es menos cierto que la ciencia es, también, un producto social. Como señala el sociólogo de la ciencia Storer (en la introducción a la recopilación de los trabajos de Merton, 1985) "concluir que el conocimiento no es modelado en absoluto por las experiencias de los hombres equivaldría a quitar a la ciencia su razón de ser, mientras que concluir que se halla totalmente modelado significarfa poner en tela de juicio, si no negar, la validez de tal conocimiento, incluyendo esta misma conclusión". Y, es que, el cientifico aislado fuera de un contexto socio-político es impensable. Los grandes investigadores han sido financiados de una manera o de otra por algún determinado poder. Existen en la historia de la ciencia decenas de ejempios que ponen de relieve tanto el desprendimiento por el amor a la ciencia como, en la mayoría de los casos, la necesidad de pasar por algún tipo de "horca caudina" a la hora de conseguir los medios necesarios para la investigación. Cajal y los Curie tuvieron que hacer filigranas para poder realizar algunas de las acciones que les llevaron a sus eminentes 
descubrimientos, indudablemente positivos para la humanidad. Además, en la actualidad, hasta los anónimos investigadores trabajan en laboratorios que han sido construidos y son mantenidos con fondos públicos o privados. Existe un claro sistema de recompensas dependientes de una serie de variables de la investigación: el apego a valores o concepciones vigentes, las necesidades puestas de relieve en un contexto y momento histórico determinado, la ruptura que supongan con lo establecido. Todo ello y mucho más está determinando las contingencias del puro hecho de la investigación.

En España, como en otros países, existen ya unas concretas líneas de investigación prioritarias establecidas por los entes públicos financiadores de la investigación. Ello ocurre, como es lógico, en mucha mayor medida en organismos privados, donde la investigación está al servicio de la industria o la tecnología.

El llamado "Documento Tres" del Ministerio de Educación y Ciencia, al establecer las plantillas de profesorado, expuso el costo relativo de las distintas enseñanzas en ciencias sociales, humanidades, carreras experimentales y tecnológicas en los distintos parses de la Comunidad Europea y otros como USA, Canadá y Japón. Pues bien, como muchos de ustedes saben, es Japón el país que más invierte en carreras "sociales". También saben ustedes que los estudios que podríamos considerar en el ámbito de las ciencias sociales cuentan con subvenciones estables muy inferiores a los estudios técnicos e igual cosa sucede en las subvenciones a la investigación.

En todo caso, parece claro que el trabajo en ciencias sociales no se prima precisamente y ello incluso aunque la calidad de vida o el estado del bienestar imperen como valores sociales y reclamos políticos.

Existen múltiples vías de comprobar las formas en las que lo social (y lo político) han mediado en la investigación cientifica derivada de los trabajos de Merton y los sociólogos de la ciencia. Sin embargo, lo que parece conveniente demostrar ahora es la escasa influencia que los hallazgos de las ciencias sociales básicas tienen sobre la planificación y programación social. Es decir, la aplicación -si la hubiera- de la ciencia social a las acciones, programas o proyectos emprendidos por los políticos.

La pregunta sería la siguiente: ¿Se reinvierten los hallazgos procedentes de la investigación social aplicándose a los problemas sociales? 0 , en otros términos, ise diseminan los resultados de la investigación social?. Weiss (1977) y Cook y cols.(1980) -entre otrosse han ocupado de este tema sistematizando diversos trabajos realizados 
con el fin de comprobar la repercusión de los hallazgos de la ciencia social en las decisiones políticas.

Si recuerdan el esquema inicialmente presentado en la primera fase del proceso de resolución de problemas sociales, el político social deberfa revisar la literatura cientifica existente sobre el problema establecido a la hora de tomar decisiones, plantear objetivos y especificar intervenciones. Asf, en el esquema que ahora ven, puede observarse un modelo (basado en Weiss, 1977) desde el cual, tras la definición del problema y el establecimiento empírico de las necesidades (que avalan su existencia), deberf́a procederse a la revisión del conocimiento científico que diera respuesta al problema planteado para, tras su analisis, tomar decisiones sobre objetivos, metas y medios que llevaran consigo, supuestamente, a la solución del problema. Y ello por cuanto, supuestamente, los hallazgos cientificos deberían avalar las decisiones políticas. Ya me he referido, anteriormente, a la importancia de la consideración de las teorfas subyacentes a las acciones emprendidas y como ello deberfa reobrar sobre la calidad de las propias decisiones sociales. Sin embargo esto no sucede. Asf, por ejemplo, el problema que conlleva la institucionalización del paciente mental se ha abordado -en algunas administraciones del Estado Español- sin tener en cuenta la investigación científica sobre el tema. En otros términos, se ha procedido a la desinstitucionalización como una meta en sí misma, sin establecer (vuelvo a repetir que, al menos, en algunos casos) sus requisitos previos: un sistema de medidas comunitarias que permitan el tratamiento sustitutivo del cliente. De no aplicar acciones en este sentido tal medida será un fracaso.

Sin embargo, esta forma de actuar no es una excepción; la falta de utilización de la investigación social parece ocurrir en parses de muy distintas latitudes geográficas y políticas, según se desprende de una serie de trabajos revisados por Weiss (1977).

Asi, Knorr (citado por Weiss 1977), tras encuestar a setenta responsables austriacos procedentes de distintos tipos de administraciones (federales, provinciales y locales) y a unos seiscientos investigadores sociales, concluyó que la utilización de los hallazgos procedentes de las ciencias sociales no sigue el mismo patrón de la diseminación de resultados procedentes de las ciencias naturales y tecnológicas. La vía de utilización parece ser indirecta, difusa y diffcil de ser localizada explícitamente.

Algo semejante parece ocurrir en los EEUU. C.aplan y cols. realizaron una entrevista desde la Universidad de Michigan a doscientos 
cuatro responsables de la política social federal. En ellos, mientras el 85\% de los encuestados estaban de acuerdo con que "las ciencias sociales pueden contribuir a efectuar una inteligente política social "y, más aún, el $83 \%$ pensaba que "la investigación social debe ser utilizada incluso aunque sus conclusiones fueran politicamente no aceptables", un $75 \%$ de los encuestados creían que "los responsables de la política social ignoraban o estaban mal informados sobre los resultados de la ciencia social si éstos no estaban en consonancia con sus creencias", y el 67\% estaban de acuerdo con que "las agencias gubernamentales tienden a ignorar los resultados de las investigaciones que no están en línea con sus asunciones y filosofías de base".

Tal vez actitudes semejantes a éstas estén presenten en algunos responsables de la política social de nuestro país cuando implantan programas sobre los que existe clara evidencia negativa en otros paises.

Un último trabajo que desearía comentar es el realizado por Weiss y Buscubalas (1980) en el que se tratan de poner de relieve los esquemas de referencia que los responsables y promotores de programas de accion social tienen a la hora de aceptar o rechazar los resultados de la investigación social. El estudio se realizó sobre 50 responsables (de 150 consultados) de acciones referidas a salud mental, alcoholismo y drogadicción. Tales responsables analizaron 50 investigaciones con base en 29 dimensiones, tales como la calidad técnica, su objetividad, su aplicabilidad, su congruencia con otros supuestos, etc.. Se obtuvieron 5 factores. El primero de ellos, denominado calidad, estaba saturado por dimensiones tales como calidad técnica, sofisticación estadística, objetividad, posibilidades de generalizacion, entre otras. Orientación a la acción fué un segundo factor, saturado por cuestiones tales como recomendaciones explícitas, variables fácilmente manipulables, implicaciones directas, aplicabilidad a otros programas en marcha, etc..El tercer factor denominado expectativas del usuario implicaba, a todas luces, la compatibilidad de los resultados de la investigación con los del responsable político. El cuarto factor fué denominado reto al "statuquo", ya que estaba en relación con el desafío que los hallazgos científicos pudieran tener sobre los valores de la institución de pertenencia. Por último, el quinto factor hacía referencia a la relevancia de la investigación para el trabajo cotidiano del posible profesional implicado.

También, se llevó a cabo un segundo análisis factorial con el fin de encontrar cuáles eran los efectos de estos factores sobre la utilidad percibida de los resultados de la investigación social. La conclusión de 
los autores es que los responsables de la polftica social encuestados utilizaron dos pruebas al objeto de discriminar la aceptación de la investigación social: una prueba de bondad y una prueba de utilidad. La prueba de bondad se basaba en dos aspectos independientes: de un lado el juicio sobre la calidad de la investigación con base en las características de rigor del método utilizado y de otro el que los resultados estuvieran de acuerdo con presupuestos previos (o conformidad). Por otra parte, la prueba de utilidad estaba apoyada sobre otros dos componentes, de un lado sobre las repercusiones prácticas de las investigaciones (orientación a la acción) y de otro sobre aquellos resultados que cuestionan los presupuestos ideológicos y los valores de la organización (o reto al statu quo).

No tenemos tiempo de comparar los resultados empíricos hallados por Weiss y Buscubalas con la tipología de Znaniecki. Como los interesados en la teorfa sobre roles sociales se habrán percatado, existe una enorme coincidencia. Roles como "asesores tecnologicos" y "creadores de conocimiento" parecen seguir existiendo en la actualidad. Con las salvedades culturales que ello debe conllevar, que el investigador social básico y aplicado conozca estos extremos pudiera ser de gran interés a la hora de realizar predicciones sobre las repercusiones de sus hallazgos en la realidad social.

En definitiva, puede concluirse que a la hora de afrontar los problemas sociales y de programar acciones que intentan su resolución los responsables de tales acciones intentan ignorar los resultados cientificos, sobre todo y fundamentalmente cuando aquéllos van en contra de sus creencias, valores o ideología. Ello pudiera deberse -como distintos autores asf lo apuntan- a diversas razones: la propia debilidad de los hallazgos procedentes de la ciencia social básica (por las razones antes dichas), el conflicto existente entre algunos de esos hallazgos y la ideología dominante, la disyunción entre la experiencia de los políticos y los resultados de los científicos.

En definitiva, como se pone de relieve en el esquema 3, los valores, la ideología política dominante influyen tanto en los "settings" sustentadores de investigación como en aquéllos a través de los que se realiza la política social. Ambos interactúan recíprocamente, aunque los políticos sociales parecen incidir más en los investigadores que viceversa asf como el estado de la zncia y el bienestar del ciudadano dependen más de los vaiores impera: s y, a través de éstos, por las subvenciones que los investigadores rec: in que por cualquier otra vía. 
Esquema 3

RELACIONES ENTRE CIENCIA Y POLTICA EN EVALUCACION DE PROGRAMAS

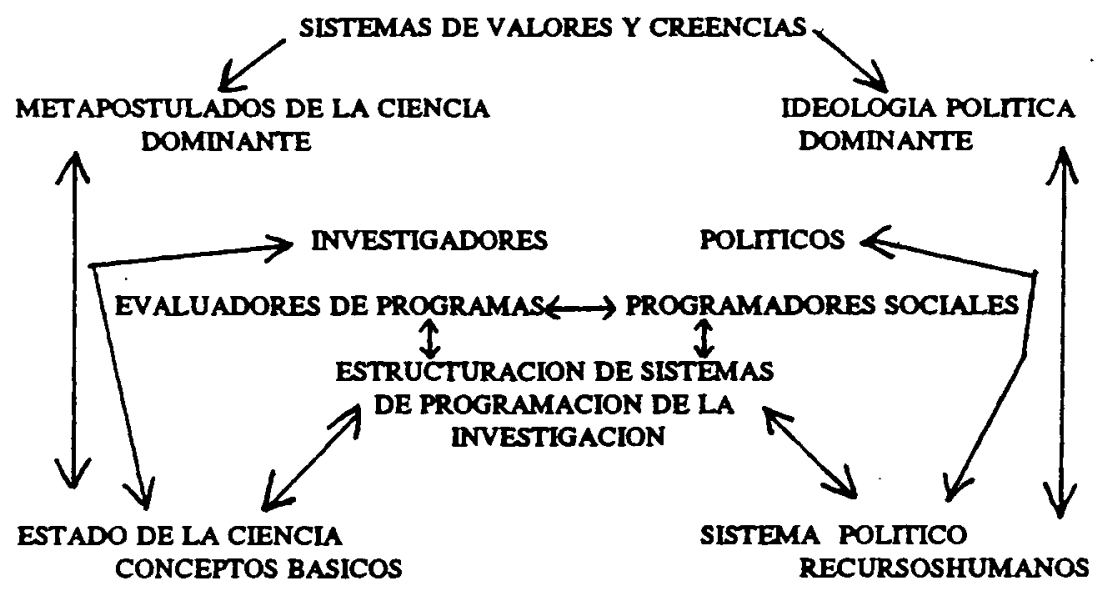

Las ideologías de la evaluación

Como señala Scriven (1983), la evaluación de programas es una de las disciplinas que, necesariamente, tiene que implicarse a si misma. Si la evaluación valora los productos sociales, en términos de acciones implantadas, la evaluación que es, desde luego, un producto social y que sólo puede tener lugar por una decisión política tendría que tomarse en serio su propia evaluación. En este caso, como en otros muchos, serfan útiles las palabras de Merton cuando, ya en 1949 -en los albores de lo que sería la Sociología de la Ciencia- decía: "los expertos en ciencias sociales han estado tan ocupados en examinar la conducta de otros que han descuidado, en gran medida, el estudio de su propia situación, sus propios problemas y su propia conducta" -y a lo que deberiamos de añadir- sus propias creencias y valores. Esto que digo no debe extrañar al auditorio cuando estamos en pleno momento de reflexión sobre lo que de social y psicológico tiene cualquier actividad humana (la sociologia de la ciencia, la psicologla social como construcción histórica, etc.). En todo caso cualquier disciplina debería conllevar unos postulados metateóricos que promovieran la reflexión entre si misma desde un contexto más amplio (asi, la sociologia de la ciencia deberfa conllevar la sociología de la sociología de la ciencia). En todo caso, siempre que se discute sobre estas cuestiones, es decir, siempre que se trata de 
realizar un análisis metateorico, recuerdo las finas reflexiones de Linton sobre la cultura y su influencia sobre la personalidad y su acertado símil sobre la dificultad que para organismos acuáticos supone la reflexión sobre la importancia que para ellos tendría el propio medio acúatico. Es decir, resulta sumamente difícil desemberazarse del contexto cultural (el agua) a la hora de analizar cualquier fenómeno social tal y como es la evaluación de programas. Ya he señalado cómo a todo lo largo del proceso de toma de decisiones, en programación y planificación social, influye lo que Mannheim considera como el sistema de creencias sobre el hombre y la sociedad. Ahora no me refiero a tal análisis ideológico en su componente político sino a aquél que acompaña a la propia actividad evaluativa. Las ideologlas a las que me voy a referir ahora suponen conceptualizaciones intermedias entre filosofías y modelos (así como los modelos son puentes entre ideologías y metodologías). En todo caso, más de una ideología puede apoyar un mismo modelo evaluativo. Según Scriven, cuatro son las ideologias más comunes subyacentes a la evaluación: la separatista, la positivista, la gerencial y la relativista.

\section{Ideología separatista}

"Yo soy un evaluador, usted es un sujeto, aquello es un objeto". Es decir, el evaluador se percibe ajeno, impolutamente no referido a la actividad que desempeña, niega o rechaza la propia implicación. Un ejemplo paradigmático de esta ideología está en los procedimientos que conlleva la evaluación de los pares (o "colegas", no en el sentido cheli del término) con el objetivo de repartir financiación de investigación o tomar decisiones sobre su futuro funcionarial por medios no calibrados, no validados y sin procedimientos de revisión. El fenómeno del cientifico acientífico, psicológicamente comprensible en términos de una epidemia evaluo-fóbica antipositivista puede representar una simple distorsión de la evaluación. El que el evaluador (o revisor), en esos ejemplos, se atribuya el don de la objetividad, es desde luego, una creencia urracional.

\section{Ideología positivista}

Esta ideología se ve asociada, cómo no, a la crítica habida en contra del positivismo a la que antes me he referido. Incluso, el trabajo cientifico es altamente evaluativo, la evaluación es frecuente y está cui- 
dadosamente dirigida por una objetividad sobrevalorada. Existen evaluadores que consideran que el producto de sus evaluaciones ha de ser entendido como "la verdad". Sin embargo, el propio Bertrand Russell comentaba: "Si un hombre os dice que posee la verdad exacta sobre algo; hay razón para creer que es un hombre equivocado: toda verdad se da siempre con un error probable". A pesar de esto, es llamativo que la ciencia del siglo XX, al menos hasta los años 60 , se presentara a sí misma libre de valores. De nuevo, como señala Scriven, se debe considerar la posibilidad de que esto forme parte, a su vez, de un sistema de creencias, generado para reducir las ansiedades de la fobia a la contaminación de los propios valores. El propio Mannheim fue criticado en este mismo sentido. En todo caso, mientras la ideología separatista rechaza la naturaleza autoreferente de la evaluación, la ideología positivista rechaza la naturaleza valorativa de la ciencia y ambas presentan inconsistencias entre los metapostulados téoricos que se profesan y la práctica profesional y ambas, según Scriven, han restringido severamente el crecimiento de la evaluación puesto que ésta viola ambos tabúes. El paradigma serfa el del cancerbero como vía para controlar al campesino pero con prohibiciones de hacer lo mismo con el castellano.

\section{Ideología gerencial}

No cabe duda de que la evaluación surge de los responsables de las decisiones sociales: políticos, legisladores, técnicos; es decir, los mismos que impulsan los programas. El evaluador depende, económicamente, de las ofertas de trabajo y éstas de los clientes, amén de que por una necesidad de autocomplacencia de los promotores, los informes favorables son más aceptados que los desfavorables. Todo ello produce un claro sesgo en la "objetividad" de la evaluación por un marcado conflicto de intereses: los del científico y el responsable político.

Ya se ha dicho, que uno de los modelos más al uso en evaluación conlleva su identificación con el cumplimento de objetivos propuestos por los responsables de los programas. A veces, como se sabe, la evaluación no sólo se realiza por iniciativa de los responsables políticos, sino que puede ser solicitada por los propios usuarios. En todo caso y generalmente, no se utilizan criterios de evaluación múltiples procedentes de los implicados en los programas.

La ideologia gerencial casa muy bien con la positivista ya que al reducir la evaluación a un concreto criterio se evita, realmente, los 
juicios de valor procedentes de múltiples fuentes criteriales.

Es el político el que señala los objetivos a alcanzar, como es el científico el que plantea la hipotesis a verificar y el evaluador el que realiza la tarea confirmatoria.

En definitiva, la evaluación de programas en las que existe la debida triangulación y se tienen en cuenta criterios múltiples a la hora de estimar la eficacia de un programa superarfa la cuestión. El planteamiento multiplista anteriormente comentado es, claramente, la alternativa de desmantelamiento de esta ideología evaluadora.

\section{Ideología relativista}

Como ya se ha dicho, mientras el positivismo cree en la existencia de un mundo externo (objetivo), algunos filosofos de la ciencia y evaluadores naturalistas tienden a alejarse de este realismo hasta llegar a un relativismo extremo lo cual, indudablemente, puede desencadenar un solipsismo estéril. Pero, además, el concepto de relativismo es refutable por propia definición: Si toda afirmación es relativa, también lo es esta afirmación. Asi, aunque parece lógico rechazar el aserto de que una sola descripción de la realidad es la correcta, no deberf́a rechazarse el supuesto de que la realidad externa existe "si muove". Aunque ésta no pueda ser exhaustivamente conocida y descrita, sf lo puede ser parcialmente y a través de múltiples prespectivas. En otras palabras, partimos de que no existe una noción exclusiva de "valor", existen múltiples criterios y prespectivas sobre lo que es valorable de un programa. Es perfectamente apropiado -según Scriven- tender a una objetividad limitada por los propios condicionantes de la investigación cientifica que se realice y, por tanto, a una objetividad relativa. La utilización de múltiples prespectivas y múltiples niveles de descripcion de un programa, abandonando cualquier asunción ingenua de que existe una sola evaluación verdadera de un programa es la única salida lógica, hoy po: hoy, ante este problema. El propio Scriven considera que el relativismo supone el más serio sesgo en evaluación de programas. Como hemos visto, del paradigma naturalista, en el que se enmarca la ide gfa relativista, emerge la asunción metodologica de que el propio eva.:. ador es el principal instrumento de la evaluación en par de igualdad con otros posibles analistas. El evaluador, es desde luego, una fuente de cuntraste más, pero una fuente de contraste cualificada que aporta métrilos y técnicas de investigación que promueven la amplificación de datos procedentes del aparato perceptivo común que permite al hombre 
el conocimiento cotidiano y que, por tanto, corrige, en alguna medida, el conocimiento subjetivo. Equiparar conocimiento cotidiano y concimiento cientifico supone negar el hecho de la propia evaluación del pensamiento humano.

Quisiera terminar sintetizando algunos de las ideas fundamentales que he tratado de exponer. No cabe duda de que la evaluación de programas es una disciplina de compromiso político y científico. Compromiso político porque el evaluador está, indudablemente, al servicio del poder civil. Su independencia no puede proceder sino de su mantenimiento en un plano de rigor metodológico y su "verdad" de la bondad o calidad con la que haya realizado su trabajo y de su fidelidad a los datos encontrados. Y, de compromiso cientifico, por cuanto el evaluador aplica los conocimientos, métodos y técnicas de investigación y porque sus esfuerzos, con muchos matices, pueden repercutir en el progreso de la ciencia que, en último extremo, debería reobrar en la solución de problemas sociales y humanos. Un progreso en esta dirección es, a todas luces, necesario, sobre todo cuando el exponencial desarrollo tecnólogico de las últimas décadas ha llevado consigo múltiples disfunciones no resueltas con la mera planificación. ¿Para cuándo dejar el empeño de un consonante y paralelo desarrollo social y humano?. La evaluación de programas puede ir en esta dirección, al menos eso es lo que yo deseo.

\section{Referencias bibliográficas}

Berk, R.A., y Rossi, P.H., 1976, Doing good or worse: evaluation research politically reexamined, Social Problems, 23, 3, pp. 337-349 Bickman, L., 1980, Using program theory in evaluation, Beverly Hills, Sage

Brown, H.J., 1984, La nueva filosofía de la ciencia, Madrid, Tecnos Campbell, D.T., 1969, Can we be scientific in applied social science?, en R.F. Conner, D.G. Altman y C. Jackson (eds.), Evaluation Studies Review Annual, Beverly Hills, Sage

Campbell, D.T., 1971, Artifact and control, en R. Rosenthal y R. L. Rosnow (eds.), Artifact in behavioral research, Nueva York, Academic

Campbell, D.T., 1977, Reforms as experiments, en G.Francis (ed.), Readings in Evaluation Research, Nueva York, Russell Sage Foundation

Caplan, N., Morrison, A. y Stambaugh, R.J., 1975, The use of social 
science knowledge in policy decision on the national level, Ann Arbor, Institute for Social Research, Universidad de Michigan Cook, T.D., 1985, Postpositist Critica Multiplims, en L.Shotland y M.M. Marck (eds.), Social science and social policy, Beverly Hills, Sage

Cook, T.D., 1986, Program evaluation: the worldly science, Annual Review of Psychology, 37, pp. 193-232

Cook, T.D., Levinson-Rose, J.G. y Pollard, W.E., 1980, The misutilization of evaluation research: some pitfalls of definition, Knowledge: creation, diffusion, utilization, 1,4 , pp. $477-498$

Cronbach, L.J., 1982, Designing evaluation of educational and social programs, Beverly Hills, Sage

Chen, H.T. y Rossi, P.H., 1980, The multi-goal theory driven approach to evaluation: a model linking basic and applied social science, Social Forces, 59, pp. 106-122

Guba, E.G., 1987, What have we learned about naturalistic evaluation? Evaluation Practice, 8, 1, pp. 23-44

Merton, R.K., 1973/1985, La sociología de la ciencia, Madrid, Alianza Universidad

Oeser, E., 1984, La evolución del método científico, en K. Lorenz y F.M. Wketits (eds.), La evolución del pensamiento, Barcelona, Argos-Vergara

Reason, P. y Rowan, J., Human Inquiry, Nueva York, Wiley

Rossi, L.M. y Freeman, F.H., 1985, Evaluation. A systematic approach, Beverly Hills, Sage

Scriven, M., 1983, Evaluation ideologies, en F. Conner, D.G. Altman y C. Jack:on (eds.), Evaluation studies review annual, Beverly Hills, Sage

Seidman, E., 1983, Unexamined pemises of social problem solving, en E. Seidman (ed.), Handbook of Social Intervention, Beverly Hills, Sage

Weiss, C., 1977, Research for policy sake: the enlightenment function of social research, Policy Analysis, 3, 4, pp. 531-545

Weiss, C., 1978, Improying the linkage between social research and public policy, en L.E. Lynn (ed.), Knowledge and policy, National Academy of Sciences, Washington, D.C.,

Weiss, C. y Buscivalas, M.J., 1981, Truth tests and utility test: a decision maker's frame of reference for social science research, en H.F. Freeman y M.A. Solomon (eds.), Evaluation studies, Vol. 3, Beverly Hills, Sage 